\title{
OPEN ACCESS

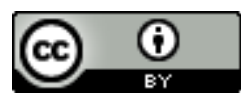 \\ PARTIAL WAVE BASIS ADAPTED TO EXTERIOR BOUNDARY CONDITIONS OF AN ELASTIC PLATE
}

\author{
Sergiu Cojocaru \\ Department of Theoretical Physics, \\ Horia Hulubei National Institute for Physics and Nuclear Engineering, \\ 077125 Magurele, Romania \\ E-mail: scojocaru@theory.nipne.ro
}

(Received November 24, 2020)

https://doi.org/10.53081/mjps.2021.20-1.02

$C Z U: 530.1+534$

\begin{abstract}
An approach to describing normal elastic vibration modes in confined systems is presented. In a standard treatment of the problem, the displacement field is represented by a superposition of partial waves of a general form, e.g., plane waves. The unknown coefficients of superposition are then obtained from the equation of motion and the full set of boundary conditions. In the proposed approach, the functional form of partial waves is chosen in such a way as to satisfy the boundary conditions on exterior surfaces identically, i.e., even if the unknown quantities determined by the remaining constraints are found in an approximation, numerically or analytically. Some examples of solutions for composite elastic plates are discussed to illustrate the efficiency of the approach and its relevance for applications.
\end{abstract}

Keywords: wave propagation, confined systems, boundary conditions

\section{Rezumat}

Este prezentată o metodă nouă pentru descrierea modurilor proprii de vibrații elastice în sisteme confinate. Într-o abordare standard a problemei, câmpul de deplasări este reprezentat de o superpoziție a undelor parțiale de formă generală, de exemplu, unde plane. Coeficienții necunoscuți ai superpoziției sunt apoi obținuți din ecuația de mișcare și din setul complet de condiții la limită. În abordarea propusă, forma funcțională a undelor parțiale este construită astfel încât condițiile la limită de pe suprafețele exterioare să fie îndeplinite în mod identic, adică chiar dacă valorile necunoscute, ce urmeaza a fi determinate de restul constrângerilor, sunt obținute într-o aproximare, numerică sau analitică. Eficiența și relevanța metodei pentru aplicații este demonstrată prin câteva exemple de soluții pentru plăci elastice compuse.

Cuvinte cheie: propagarea undelor, sisteme confinate, condiții la limită 


\section{Introduction}

We consider an infinite composite plate with layers of homogeneous materials labeled with index $\gamma=1,2, \ldots$ (e.g., layer thickness $h_{\gamma}$ ) and briefly outline the general formalism [e.g., 1-5]. The stress-strain relation in a given material is as follows:

$$
\Sigma_{\mathrm{ij}, \gamma}=\lambda_{\gamma} \delta_{\mathrm{ij}} \varepsilon_{\mathrm{kk}}+2 \mu_{\gamma} \varepsilon_{\mathrm{ij}}
$$

where $\delta_{\mathrm{ij}} \quad$ is the Kronecker symbol, $i=1,2,3$, double index implies summation, $\lambda, \mu$ are the two Lamé stiffness parameters and the strain tensor at a given point $\boldsymbol{r}=\left(x_{1}=x, x_{2}=y, x_{3}=z\right)$ and time $t$ is defined by respective space derivatives of the displacement field $U(r, t)$ components

$$
\varepsilon_{\mathrm{km}}(r, t)=\left(\frac{\partial U_{k}}{\partial x_{m}}+\frac{\partial U_{m}}{\partial x_{k}}\right) / 2 .
$$

Solutions of elastodynamic equations

$$
\rho \frac{\partial^{2} U_{i}(r, t)}{\partial t^{2}}=\frac{\partial \Sigma_{\mathrm{ij}}(r, t)}{\partial r_{j}}
$$

are represented by a superposition of normal modes propagating in-plane with the wave vector $\boldsymbol{q}_{\|}$ and indexed by $n=1,2, \ldots$ :

$$
u_{n}\left(z, q, \omega_{n}(q)\right) \exp \left(\mathrm{iqx}-i \omega_{n} t\right) \frac{\mathrm{dq}}{2 \pi}
$$

Here we have set the $x$ - axis collinear to the wave vector, so that $r q_{\|}=q_{x} x+q_{y} y=q_{x}$ and plate thickness is spanned by the $z$ - axis. Equations are then decoupled according to the polarization pattern into the shear horizontal, $u=\left(0, u_{y}, 0\right)$, and mixed (shear vertical and longitudinal [6]), $u=\left(u_{x}, 0, u_{z}\right)$. The latter, which are referred to as Rayleigh-Lamb waves, are examined in the following. The relevant components of the stress tensor are as follows:

$$
\begin{gathered}
i \sigma_{\mathrm{zz}}(z)=\left(\lambda_{\gamma}+2 \mu_{\gamma}\right) \frac{\partial\left(i u_{z}\right)}{\partial z}-\lambda_{\gamma} q u_{x}, \\
\sigma_{\mathrm{xz}}(z)=\mu_{\gamma}\left(\frac{\partial u_{x}}{\partial z}+q\left(i u_{z}\right)\right) .
\end{gathered}
$$

From Eq. (1) we then obtain for the mode amplitudes 


$$
\begin{aligned}
& s_{\gamma}^{2} \frac{\partial^{2} u_{x, \gamma}}{\partial z^{2}}-\ell_{\gamma}^{2} q^{2} w_{\gamma}^{2} u_{x, \gamma}+\left(\ell_{\gamma}^{2}-s_{\gamma}^{2}\right) q \frac{\partial \mathrm{iu}_{z, \gamma}}{\partial z}=0, \\
& \ell_{\gamma}^{2} \frac{\partial^{2} i u_{z, \gamma}}{\partial z^{2}}-s_{\gamma}^{2} q^{2} v_{\gamma}^{2} \mathrm{iu}_{z, \gamma}-\left(\ell_{\gamma}^{2}-s_{\gamma}^{2}\right) q \frac{\partial u_{x, \gamma}}{\partial z}=0,
\end{aligned}
$$

where $\ell=\sqrt{(\lambda+2 \mu) / \rho}, s=\sqrt{\mu / \rho}$ are the bulk longitudinal and transverse sound velocities in the respective materials of mass density $\rho$. Quantities $v_{\gamma}$ and $w_{\gamma}$ defined as

$$
w_{\gamma}=\sqrt{1-\left(c / \ell_{\gamma}\right)^{2}}, v_{\gamma}=\sqrt{1-\left(c / s_{\gamma}\right)^{2}} .
$$

contain phase velocities $c=\omega / q$ of normal modes ( $n$ index is dropped).

\section{Basis Set Functions}

Equations (2) are to be solved by specifying the displacement and/or stress fields at the external boundaries, $z-d_{\gamma}=0$. In addition, these fields are subject to continuity conditions at the interfaces between different media: for a double layer, the interface is chosen at $z=0$, so that the upper bound is at $z=d_{1}=h_{1}$ and the lower bound at $\quad z=d_{2}=-h_{2}$. The standard approach is to use a universal basis set of incoming and outgoing plane waves, which does not depend on details of the structure. It is well suited for treating systems embedded in an infinite medium, and the problem is then mapped into one of a linear algebra for the expansion coefficients by applying the complete set of boundary conditions. Dispersion curves are obtained from the respective secular equation in an analytical or numerical approximation. The proposed alternative necessarily produces the same frequency spectrum; however, the basis set functions will not be universal, since their functional form is required to transform some of the boundary conditions into identities. To this end, equations defining the exterior surfaces are introduced as arguments of the basis set functions. In this case, exterior boundary conditions allow reducing the number of independent coefficients by the number of constraint equations. As a consequence, the expressions of the wave fields in terms of these independent algebraic variables automatically satisfy the above boundary conditions.

For flat boundaries, we can start with the following general form of the partial wave superposition:

$$
\begin{aligned}
& u_{x, \gamma}=\xi_{w, \gamma} \sinh \left(\left(z-d_{\gamma}\right) \mathrm{qw}_{\gamma}\right)+\xi_{v, \gamma} \sinh \left(\left(z-d_{\gamma}\right) \mathrm{qv}_{\gamma}\right)+\theta_{w, \gamma} \cosh \left(\left(z-d_{\gamma}\right) \mathrm{qw}_{\gamma}\right) \\
& +\theta_{v, \gamma} \cosh \left(\left(z-d_{\gamma}\right) \mathrm{qv}_{\gamma}\right) . \\
& \mathrm{iu}_{z, \gamma}=\zeta_{w, \gamma} \sinh \left(\left(z-d_{\gamma}\right) \mathrm{qw}_{\gamma}\right)+\zeta_{v, \gamma} \sinh \left(\left(z-d_{\gamma}\right) \mathrm{qv}_{\gamma}\right)+\eta_{w, \gamma} \cosh \left(\left(z-d_{\gamma}\right) \mathrm{qw}_{\gamma}\right) \\
& +\eta_{v, \gamma} \cosh \left(\left(z-d_{\gamma}\right) \mathrm{qv}_{\gamma}\right) .
\end{aligned}
$$


It is evident that the substitution of these expressions into the governing equations (2) reduces the number of variables by half (in what follows, the $\gamma$ index identifying the layers will be occasionally dropped for brevity):

$$
\eta_{w}=w \xi_{w}, \eta_{v}=\xi_{v} / v, \theta_{w}=\zeta_{w} / w, \theta_{v}=\zeta_{v} v
$$

By introducing the new notations

$$
X=\xi_{v}, Y=\theta_{v}, R=\xi_{w}, S_{\gamma}=\theta_{w},
$$

we get the final general form of the partial wave expansion:

$$
\begin{aligned}
& u_{x, \gamma}=X_{\gamma} \sinh \left(\left(z-d_{\gamma}\right) \mathrm{qv}_{\gamma}\right)+Y_{\gamma} \cosh \left(\left(z-d_{\gamma}\right) \mathrm{qv}_{\gamma}\right) \\
& +S_{\gamma} \cosh \left(\left(z-d_{\gamma}\right) \mathrm{qw}_{\gamma}\right)+R_{\gamma} \sinh \left(\left(z-d_{\gamma}\right) \mathrm{qw}_{\gamma}\right) \\
& \mathrm{iu}_{z, \gamma}=\frac{1}{v_{\gamma}}\left[X_{\gamma} \cosh \left(\left(z-d_{\gamma}\right) \mathrm{qv}_{\gamma}\right)+Y_{\gamma} \sinh \left(\left(z-d_{\gamma}\right) \mathrm{qv}_{\gamma}\right)\right] \\
& +w_{\gamma}\left[R_{\gamma} \cosh \left(\left(z-d_{\gamma}\right) \mathrm{qw}_{\gamma}\right)+S_{\gamma} \sinh \left(\left(z-d_{\gamma}\right) \mathrm{qw}_{\gamma}\right)\right] \\
& \frac{i \sigma_{\mathrm{zz}, \gamma}(q, z)}{2 \mu_{\gamma} q}=X_{\gamma} \operatorname{sinhqv}_{\gamma}\left(z-d_{\gamma}\right)+Y_{\gamma} \operatorname{coshqv} \gamma\left(z-d_{\gamma}\right) \\
& +\frac{\left(1+v_{\gamma}^{2}\right)}{2}\left[R_{\gamma} \operatorname{sinhqw_{\gamma }}\left(z-d_{\gamma}\right)+S_{\gamma} \cosh \left(\left(z-d_{\gamma}\right) \mathrm{qw}_{\gamma}\right)\right] \\
& \frac{v_{\gamma} \sigma_{\mathrm{xz}, \gamma}(q, z)}{\left(1+v_{\gamma}^{2}\right) \mu_{\gamma} q}=X_{\gamma} \operatorname{coshqv}_{\gamma}\left(z-d_{\gamma}\right)+Y_{\gamma} \operatorname{sinhqv}_{\gamma}\left(z-d_{\gamma}\right) \\
& +\frac{2 v_{\gamma} w_{\gamma}}{\left(1+v_{\gamma}^{2}\right)}\left[R_{\gamma} \operatorname{coshqw}_{\gamma}\left(z-d_{\gamma}\right)+S_{\gamma} \operatorname{sinhqw}_{\gamma}\left(z-d_{\gamma}\right)\right] .
\end{aligned}
$$

We have also used the following identity:

$$
\ell_{\gamma}\left(1-w_{\gamma}^{2}\right)=s_{\gamma}\left(1-v_{\gamma}^{2}\right)
$$

\section{Traction-Free Plate}

To illustrate the procedure, we first consider the case of a traction-free composite plate. Relations that come from the zero stress requirement $\sigma\left(z=d_{\beta}\right)=0$ reduce the number of variables by four when applied to the general equations $(5,6)$ 


$$
S_{\beta}=-Y_{\beta} \frac{2}{1+v_{\beta}^{2}}, R_{\beta}=-X_{\beta} \frac{1+v_{\beta}^{2}}{2 v_{\beta} w_{\beta}},
$$

here, $\gamma=\beta=1,2=$ top, bottom . Note that if we consider a bilayered plate, then $S, R$ variables are completely removed from consideration and we are left with only $\left\{X_{\gamma}, Y_{\gamma}\right\}$ as unknowns. It can then be verified that, in terms of these variables, the traction-free BC are indeed satisfied automatically:

$$
\begin{aligned}
& u_{x}(z)=X_{\gamma}\left(\sinh \left(\left(z-d_{\gamma}\right) \mathrm{qv}_{\gamma}\right)-\frac{\left(1+v_{\gamma}^{2}\right)}{2 v_{\gamma} w_{\gamma}} \sinh \left(\left(z-d_{\gamma}\right) \mathrm{qw}_{\gamma}\right)\right) \\
& +Y_{\gamma}\left(\cosh \left(\left(z-d_{\gamma}\right) \mathrm{qv}_{\gamma}\right)-\frac{2}{\left(1+v_{\gamma}^{2}\right)} \cosh \left(\left(z-d_{\gamma}\right) \mathrm{qw}_{\gamma}\right)\right), \\
& \mathrm{iu}_{z}(z)=\frac{1}{v_{\gamma}} X_{\gamma}\left(\cosh \left(\left(z-d_{\gamma}\right) \mathrm{qv}_{\gamma}\right)-\frac{\left(1+v_{\gamma}^{2}\right)}{2} \cosh \left(\left(z-d_{\gamma}\right) \mathrm{qw}_{\gamma}\right)\right) \\
& +\frac{1}{v_{\gamma}} Y_{\gamma}\left[\sinh \left(\left(z-d_{\gamma}\right) \mathrm{qv}_{\gamma}\right)-\frac{2 w_{\gamma} v_{\gamma}}{\left(1+v_{\gamma}^{2}\right)} \sinh \left(\left(z-d_{\gamma}\right) \mathrm{qw}_{\gamma}\right)\right], \\
& \frac{i \sigma_{\mathrm{zz}}(z)}{2 \mu_{\gamma} q}=X_{\gamma}\left(\sinh \left(\left(z-d_{\gamma}\right) \mathrm{qv}_{\gamma}\right)-\frac{\left(1+v_{\gamma}^{2}\right)^{2}}{4 v_{\gamma} w_{\gamma}} \sinh \left(\left(z-d_{\gamma}\right) \mathrm{qw}_{\gamma}\right)\right) \\
& +Y_{\gamma}\left(\cosh \left(\left(z-d_{\gamma}\right) \mathrm{qv}_{\gamma}\right)-\cosh \left(\left(z-d_{\gamma}\right) \mathrm{qw}_{\gamma}\right)\right), \\
& \frac{v_{\gamma} \sigma_{\mathrm{xz}}(z)}{\left(1+v_{\gamma}^{2}\right) \mu_{\gamma} q}=X_{\gamma}\left(\cosh \left(\left(z-d_{\gamma}\right) \mathrm{qv}_{\gamma}\right)-\cosh \left(\left(z-d_{\gamma}\right) \mathrm{qw}_{\gamma}\right)\right) \\
& +Y_{\gamma}\left(\sinh \left(\left(z-d_{\gamma}\right) \mathrm{qv}_{\gamma}\right)-\frac{4 v_{\gamma} w_{\gamma}}{\left(1+v_{\gamma}^{2}\right)^{2}} \sinh \left(\left(z-d_{\gamma}\right) \mathrm{qw}_{\gamma}\right)\right) \text {. }
\end{aligned}
$$

The physical meaning of variables $\left\{X_{\beta}, Y_{\beta}\right\}$ is that they are proportional to the components of the displacement field on the outer surfaces. The secular equation is then a result of matching the continuity of the four fields in Eqs. (7)-(10) at the interface $z=0$. It can be verified that the complete set of equations, including normalization of the amplitudes, coincides with those obtained in [7]. If a plate is composed of a larger number of materials, then the inner layers will be subject to continuity conditions only and the $R_{\gamma}$ and $S_{\gamma}$ variables for $\gamma \neq \beta$ will be included.

It is also instructive to see how Lamb equations for a homogeneous free plate and their symmetry properties emerge as a limiting case of the obtained expressions, $\beta=1,2$. To avoid lengthier arguments, we take $d_{1}=-d_{2}=h$ so that the interface $\quad z=0$ is at the middle plane. 
In this case, equations resulting from continuity of (7)-(10) are decoupled into a $2 \times 2$ pair with symmetric and antisymmetric combinations of variables: $\left\{X_{2}+X_{1}, Y_{2}-Y_{1}\right\}$ for Eqs. $(7,9)$ and $\left\{X_{2}-X_{1}, Y_{2}+Y_{1}\right\}$ for Eqs. $(8,10)$. Namely,

$$
\begin{array}{r}
\left(X_{2}+X_{1}\right)\left(\sinh (\text { hqv })-\frac{\left(1+v^{2}\right)}{2 v w} \sinh (\text { hqw })\right) \\
+\left(Y_{2}-Y_{1}\right)\left(\cosh (\text { hqv })-\frac{2}{\left(1+v^{2}\right)} \cosh (\text { hqw })\right)=0,
\end{array}
$$

$\mu q\left(\left(X_{2}+X_{1}\right)\left(\sinh (\right.\right.$ hqv $)-\frac{\left(1+v^{2}\right)^{2}}{4 v w} \sinh ($ hqw $\left.)\right)+\left(Y_{2}-Y_{1}\right)(\cosh ($ hqv $)-\cosh ($ hqw $\left.))\right)=0$ and

$$
\begin{gathered}
\frac{\left(X_{2}-X_{1}\right)}{v}\left(\cosh (\text { hqv })-\frac{\left(1+v^{2}\right)}{2} \cosh (\text { hqw })\right) \\
+\frac{\left(Y_{2}+Y_{1}\right)}{v}\left(\sinh (\text { hqv })-\frac{2 w v}{\left(1+v^{2}\right)} \sinh (\text { hqw })\right)=0, \\
\frac{\mu q\left(1+v^{2}\right)}{v}\left(\left(X_{2}-X_{1}\right)(\cosh (\text { hqv })-\cosh (\text { hqw }))\right. \\
\left.+\left(Y_{2}+Y_{1}\right)\left(\sinh (\text { hqv })-\frac{4 v w}{\left(1+v^{2}\right)^{2}} \sinh (\text { hqw })\right)\right)=0 .
\end{gathered}
$$

Solutions are given by equating the product of the two determinants to zero. It is obvious the two systems of equations above are different and can only produce the same eigenvalues at the points of intersection between the two corresponding sets of dispersion curves. Therefore, nontrivial solutions of the secular equation, i.e., the whole set of four equations, are generally found when one of the $2 \times 2$ pairs has a non-trivial and the other has a trivial solution. This gives only two possibilities: $\left\{X_{2}=X_{1}, Y_{2}=-Y_{1}\right\} \quad$ (A) and $\left\{X_{2}=-X_{1}, Y_{2}=Y_{1}\right\}$ (S). In this case, we find for the case $(A)$ :

$$
\frac{\mu q\left(1-v^{2}\right)}{\mathrm{Vw}\left(1+v^{2}\right)}\left(4(\mathrm{vw}) \cosh (\mathrm{hqw}) \sinh (\mathrm{hqv})-\left(1+v^{2}\right)^{2} \sinh (\mathrm{hqw}) \cosh (\mathrm{hqv})\right)=0
$$

and for the case $(\mathrm{S})$

$$
\frac{\mu q\left(1-v^{2}\right)}{v^{2}\left(1+v^{2}\right)}\left(4(\mathrm{vw}) \cosh (\mathrm{hqv}) \sinh (\mathrm{hqw})-\left(1+v^{2}\right)^{2} \sinh (\mathrm{hqv}) \cosh (\mathrm{hqw})\right)=0 .
$$


These expressions are the well-known Lamb equations. Their transformation properties with respect to reflection in the $z-$ plane are then easily obtained from the explicit form of the fields in $(7)-(10)$ :

$$
\begin{gathered}
\left\{X_{2}=X_{1}, Y_{2}=-Y_{1}\right\}_{A}: u_{x}(z)=-u_{x}(-z), \sigma_{\mathrm{zz}}(z)=-\sigma_{\mathrm{zz}}(-z) ; \\
u_{z}(z)=u_{z}(-z), \sigma_{\mathrm{xz}}(z)=\sigma_{\mathrm{xz}}(-z) . \\
\left\{X_{2}=-X_{1}, Y_{2}=Y_{1}\right\}_{S}: u_{x}(z)=u_{x}(-z), \sigma_{\mathrm{zz}}(z)=\sigma_{\mathrm{zz}}(-z) ; \\
u_{z}(z)=-u_{z}(-z), \sigma_{\mathrm{xz}}(z)=-\sigma_{\mathrm{xz}}(-z) .
\end{gathered}
$$

Thus, we have derived the transformation properties of the Lamb modes directly from our approach instead of introducing symmetry relations $(11,12)$ from the outset, based on general theorems, in order to derive the Lamb equations in the end.

\section{Clamped Plate}

To illustrate the approach using another example, let us now examine a bilayered plate clamped at the top and bottom surfaces, $\gamma=\beta$. From zero-displacement condition applied to the general expressions in (3) and (4) we find:

$$
S_{\beta}=-Y_{\beta}, R_{\beta}=-X_{\beta} \frac{1}{v_{\beta} w_{\beta}} .
$$

It can be seen again that the exterior boundary conditions at $z=d_{\gamma}$ are accounted for by our basis set.

$$
\begin{aligned}
& u_{x}(z)=X_{\gamma}\left(\sinh \left(\left(z-d_{\gamma}\right) \mathrm{qv}_{\gamma}\right)-\frac{1}{v_{\gamma} w_{\gamma}} \sinh \left(\left(z-d_{\gamma}\right) \mathrm{qw}_{\gamma}\right)\right) \\
& +Y_{\gamma}\left(\cosh \left(\left(z-d_{\gamma}\right) \mathrm{qv}_{\gamma}\right)-\cosh \left(\left(z-d_{\gamma}\right) \mathrm{qw}_{\gamma}\right)\right), \\
& \mathrm{iu}_{z}(z)=\frac{1}{v_{\gamma}} X_{\gamma}\left(\cosh \left(\left(z-d_{\gamma}\right) \mathrm{qv}_{\gamma}\right)-\cosh \left(\left(z-d_{\gamma}\right) \mathrm{qw}_{\gamma}\right)\right) \\
& +\frac{1}{v_{\gamma}} Y_{\gamma}\left(\sinh \left(\left(z-d_{\gamma}\right) \mathrm{qv}_{\gamma}\right)-v_{\gamma} w_{\gamma} \sinh \left(\left(z-d_{\gamma}\right) \mathrm{qw}_{\gamma}\right)\right),
\end{aligned}
$$




$$
\begin{gathered}
\frac{i \sigma_{\mathrm{zz}}(z)}{2 \mu_{\gamma} q}=X_{\gamma}\left(\sinh \left(\left(z-d_{\gamma}\right) \mathrm{qv}_{\gamma}\right)-\frac{\left(1+v_{\gamma}^{2}\right)}{2 v_{\gamma} w_{\gamma}} \sinh \left(\left(z-d_{\gamma}\right) \mathrm{qw}_{\gamma}\right)\right) \\
+Y_{\gamma}\left(\cosh \left(\left(z-d_{\gamma}\right) \mathrm{qv}_{\gamma}\right)-\frac{\left(1+v_{\gamma}^{2}\right)}{2} \cosh \left(\left(z-d_{\gamma}\right) \mathrm{qw}_{\gamma}\right)\right) \\
\frac{v_{\gamma} \sigma_{\mathrm{xz}}(z)}{\left(1+v_{\gamma}^{2}\right) \mu_{\gamma} q}=X_{\gamma}\left(\cosh \left(\left(z-d_{\gamma}\right) \mathrm{qv}_{\gamma}\right)-\frac{2}{\left(1+v_{\gamma}^{2}\right)} \cosh \left(\left(z-d_{\gamma}\right) \mathrm{qw}_{\gamma}\right)\right) \\
+Y_{\gamma}\left(\sinh \left(\left(z-d_{\gamma}\right) \mathrm{qv}_{\gamma}\right)-\frac{2 v_{\gamma} w_{\gamma}}{\left(1+v_{\gamma}^{2}\right)} \sinh \left(\left(z-d_{\gamma}\right) \mathrm{qw}_{\gamma}\right)\right)
\end{gathered}
$$

Similarly to the case of a free plate, the final equations are obtained from continuity of the fields in the above expressions at the internal boundary $z=0$. Likewise, the single layer limit leads to the decoupling of the secular equation into a $2 \times 2$ pair corresponding to $\mathrm{S}$ (Eq. (12)), and A modes (Eq. (11)), their spectra being determined by

$$
\mu q \frac{1-v^{2}}{v^{2}}(\cosh (\mathrm{hqw}) \sinh (\mathrm{hqv})-(\mathrm{vw}) \sinh (\mathrm{hqw}) \cosh (\mathrm{hqv}))=0
$$

(A)

$$
\mu q \frac{1-v^{2}}{\mathrm{vw}}(\cosh (\mathrm{hqv}) \sinh (\mathrm{hqw})-(\mathrm{vw}) \sinh (\mathrm{hqv}) \cosh (\mathrm{hqw}))=0
$$

These are essentially the same secular equations as analyzed in [8]. On the other side, as noted above, our expressions for the wave amplitudes appear to differ from those of a standard representation. However, it can be shown that, for the frequencies coinciding with eigenvalues of the secular equations, the amplitudes become equivalent to the standard form.

\section{Summary}

The main advantage of the approach is that it offers a simple and direct route to the irreducible form of the secular equation. This simplification has allowed to obtain a complete analytic solution describing the long wavelength region of the vibration spectrum [7] of a twolayered system. In this case, the set of independent material parameters is 8-dimensional: the longitudinal and transverse sound velocities in the two media, respective mass densities and thicknesses of the layers. These systems are quite common in a broad range of applications; 
however, experimental or numerical exploration of the full parametric space is hopeless in view of understanding and engineering of their properties. Nevertheless, the above approach reveals an unexpected evolution with the layer thickness of the slowest (flexural) mode of the velocity spectrum and other features [9], which could be exploited for specific purposes. The approach also provides an interesting new perspective of extension to confined systems of different geometries. It may also be of interest in implementing various approximation schemes, since, unlike other approaches, some of the boundary conditions are satisfied identically from outset and approximation errors can only show up, e.g., in a mismatch of the fields at interfaces.

Acknowledgments. This work was financially supported by ANCS Romania (project no. PN 19060101/2019-2022).

\section{References}

[1] S. Cojocaru, Mold. J. Phys. Sci. 17, 75 (2018).

[2] B. A. Auld, Acoustic Fields and Waves in Solids, 2nd ed., Krieger, Malabar, FL, 1990.

[3] L. M. Brekhovskikh and O. A. Godin, Acoustics of Layered Media I, Springer, New York, 1990.

[4] J. L. Rose, Ultrasonic Waves in Solid Media, Cambridge University Press, Cambridge, 2004.

[5] J. L. Rose, Ultrasonic Guided Waves in Solid Media, Cambridge University Press, New York, 2014).

[6] H. Ezawa, Ann. Phys. (N.Y.) 67, 438 (1971).

[7] S. Cojocaru, Wave Motion 92, 102430 (2020).

[8] E. Kausel, P. Malischewsky, and J. Barbosa, Wave Motion 56, 22 (2015).

[9] S. Cojocaru, Unusual Size Dependence of Acoustic Properties in Layered Nanostructures, In:

I. Tiginyanu, V. Sontea, and S. Railean (Eds.) 4th Int. Conf. on Nanotechnologies and Biomedical Engineering, ICNBME 2019, IFMBE Proc., vol 77, Springer, Cham.

https://doi.org/10.1007/978-3-030-31866-6_5 